\title{
EFEITO DO MÉTODO BOARD FITNESS NA COORDENAÇÃO MOTORA DE MULHERES ATIVAS
}

Rafael Cesar Ferrari dos Santos, Janaina Pereira Duarte Bezerra.

Universidade do Oeste Paulista - UNOESTE, Curso de Educação Física, Presidente Prudente, SP. E-mail: Rafael.edfisica1@gmail.com

Programa Especial de Iniciação Científica - PEIC

\section{RESUMO}

Estudos mostram que na fase adulta, o desenvolvimento da coordenação motora, especificamente o equilíbrio corporal não estimulado se mantem estável, podendo até regredir com o passar dos anos, tendo um maior declínio na terceira idade. O objetivo foi verificar o efeito de um programa de treinamento com a metodologia Board Fitness, sobre o possível ganho do equilíbrio corporal em mulheres adultas ativas. A metodologia da pesquisa foi empírica de caráter quantitativa. Aplicou-se o teste "parada da cegonha" antes e após o treinamento, foram realizadas aulas durante oito semanas do método Board Fitness, sendo duas vezes por semana, com duração de 30 minutos. Antes do treinamento a média foi de 2,6 segundos de equilíbrio corporal, e após obtevese a média de 8 segundos, demonstra o avanço na classificação do nível de equilíbrio corporal de iniciante para intermediário. $O$ treinamento Board Fitness contribui para a melhora do aspecto equilíbrio em mulheres adultas.

Palavras-chave: Equilíbrio Postural, Educação Física e Treinamento, Desempenho motor, Teste motor, Saúde da mulher.

\section{EFFECT OF THE BOARD FITNESS METHOD ON MOTOR COORDINATION OF ACTIVE WOMEN}

\begin{abstract}
Studies show that in adulthood, the development of motor coordination, specifically the unstimulated body balance, remains stable and may even regress over the years, with a greater decline in the elderly. The objective was to verify the effect of a training program with the Board Fitness methodology on the possible gain of body balance in active adult women. The methodology of the research was quantitative empirical. The "Stork Parade" test was applied before and after the training, during eight weeks of the Board Fitness method, being twice a week, lasting 30 minutes. Before the training the mean was 2.6 seconds of body balance, and after a mean of 8 seconds, it shows the progress in the classification of the body balance level from beginner to intermediate. Board Fitness training contributes to improved balance in adult women. Keywords: Postural Balance, Physical Education and Training, Motor Performance, Motor Testing, Women's Health.
\end{abstract}




\section{INTRODUÇÃO}

O significado da coordenação motora é a organização de execução de movimento visando o objetivo pré-estabelecido. Para isso dependemos da harmonização de todas as forças internas e externas que surgem e suas constantes mudanças de grandeza'.

À medida que entramos na idade adulta, experimentamos algumas alterações fisiológicas e físicas que afetam em nosso comportamento motor. Do mesmo modo, quando entramos nesse ciclo da vida, aparecem modificações em nossas habilidades afetivas e cognitivas que alteram a maneira de reagirmos ao nosso ambiente. Essas modificações ficam evidentes quando um indivíduo mais velho compensa uma desaceleração no seu tempo de reação, usando estratégias cognitivas diferentes para realizar suas tarefas ${ }^{2}$.

Com a chegada à fase adulta o processo da manutenção do equilíbrio e de controle postural torna-se menos eficiente, porém a reação de ativação muscular na recuperação do equilíbrio em adultos jovens tende a ser mais rápida que em adultos mais velhos, por tanto quanto antes a manutenção no equilíbrio corporal através da atividade física regular, maior qualidade de vida para a velhice nas atividades do cotidiano e até na locomoção².

Atualmente existem diversos estilos e modalidade de exercícios físicos, e cada um tem seu objetivo, e procura gerar seus benefícios. Dentre esses, apresentamos método Board Fitness. Esse estilo de exercício mistura movimentos que são praticados com o equipamento semelhante a um skate e exercícios funcionais, trazendo uma melhora no condicionamento e nas capacidades funcionais, e assim melhorando a coordenação motora ${ }^{3}$.

O Funcional Board Fitness foi criado por Mateus Benelli e patenteado no ano de 2010. O objetivo dessa modalidade é promover o condicionamento físico trazendo mudanças estéticas relacionados ao emagrecimento. São utilizados inúmeros exercícios multi-planares e multiarticulares com intercalação de momentos que usam contração isotônica e isométrica nos seus diferentes segmentos. Os movimentos são na sua maioria feitos em grande amplitude articular para ter maior gasto energético possível. Sua metodologia traz uma alta intensidade de esforço, e a aula é de 30 minutos e com opção de 45 minutos $^{4}$.

Sendo assim, a prática de novas modalidades, como a do método Board Fitness, tem tido uma aderência significativa de participantes, isso se justifica por ser traduzida como uma ferramenta que potencializa a saúde, através da melhora das capacidades físicas dos sujeitos. Entretanto, na literatura científica identificamos uma escassez de estudos que tem por objetivo verificar se a prática de diferentes modalidades físicas contribui para o desenvolvimento motor de adultos.

Por outro lado, estudos da área do desenvolvimento humano, no que diz respeito ao desenvolvimento motor de adultos não tem sido uma preocupação pelos profissionais da área da Educação Física, que acabam centrando seus estudos geralmente na fase da infância e da terceira idade.

Nesse sentido, o presente estudo torna-se relevante no sentido de investigar se a prática do método Board Fitness pode ser recomendada para sujeitos que necessitam aprimorar sua coordenação motora, enfatizando o aspecto equilíbrio corporal, assim como, de destacarmos a necessidade de estudos na área do desenvolvimento motor focando a fase adulta do desenvolvimento humano.

Diante disso, o objetivo do estudo foi verificar o efeito do método Board Fitness em mulheres ativas na coordenação motora, especificamente do equilíbrio corporal.

\section{METODOLOGIA}

O estudo foi empírico de abordagem quantitativo com mulheres praticantes de Board Fitness. A pesquisa foi submetida ao Comitê de Ética em Pesquisa da Universidade do Oeste Paulista UNOESTE, para avaliação antes de sua implementação. Número do cep: 56940116.7.0000.5515. 
Os critérios de inclusão estabelecidos foram: ser do sexo feminino, terem idade entre 20 a 45 anos; estarem aptas fisicamente para participar do estudo experimental; praticantes de atividade física regulares há mais de um ano. Os critérios de exclusão adotados foram: Falta de capacidade para responder ao questionário avaliativo e frequência inferior a 85\% no programa.

As participantes foram avaliadas entre os meses de setembro e outubro de 2016. Realizando o treinamento por 8 semanas, com frequência de 2 vezes cada semana. Foi aplicado em uma academia na cidade de Presidente Bernardes - SP. As mulheres foram convidadas pessoalmente à participarem da investigação, 5 participantes frequentavam a academia, 5 não frequentavam a academia, porém praticavam exercícios físico.

Foram incluídas no estudo mulheres ativas, para comprovação foi aplicado o questionário IPAQ- Questionário Internacional de Atividade Física. Três níveis de atividade física são propostos: baixo/inativo, moderado e alto. Participaram da pesquisa aquelas que tiverem o índice de moderado ou alto de atividade física.

Para verificar o nível de equilíbrio corporal antes e após o treinando de Board Fitness, aplicou-se o teste "parada da cegonha" ${ }^{6}$. O teste foi feito na posição em pé, onde o avaliado permaneceu apoiado em um dos pés, sendo aquele que possui mais segurança, com as mãos apoiadas na altura da cintura e com o outro pé apoiado, com a face plantar, na parte interna do joelho dominante. Após o sinal do avaliador, a participante elevou o calcanhar e permaneceu em apoio sobre os artelhos, ao perder o equilíbrio tocando os calcanhares no chão ou tirando as mãos da cintura, o teste foi interrompido pelo avaliador. Foram realizadas três tentativas com os olhos abertos e três tentativas com os olhos fechados, neste caso a abertura dos olhos o teste também foi interrompido. Com o cronômetro o resultado é aferido em segundos com números inteiros, sendo que houve arredondamento para mais ou para menos, dependendo da fração decimal se aproximar até 0,5 ou maior que 0,5 respectivamente. Por fim, foi considerado o valor da melhor tentativa executada nas três repetições, como resultado do teste.

O teste classifica o desempenho em 5 níveis, como nos mostra a tabela abaixo.

Tabela 1. Normas em segundos para a Parada da Cegonha.

\begin{tabular}{lc}
\hline Nível de Desempenho & Mulheres \\
\hline Avançado & 28 ou mais \\
Intermediário Avançado & $23-27$ \\
Intermediário & $8-22$ \\
Iniciante Avançado & $3-7$ \\
Iniciante & $0-2$ \\
\hline
\end{tabular}

Fonte: Tritschler (2003)

As aulas foram sistematizadas por meio do protocolo do método Board Fitness (BENELLI, 2014). O treinamento é composto por diferentes tipos de exercícios, como: afundo, agachamento, abdominais, ponte, prancha.

Em seu sistema, consiste em planejar uma aula de 30 minutos: um aquecimento e, 4 treinos, contendo nos treinos 4 exercícios de cada segmento corporal.

Após o fim do treinamento, no período de oito semanas foi aplicado novamente o teste de equilíbrio corporal nas mesmas condições da aplicação do primeiro teste, para a verificação da possível melhora no equilíbrio corporal. 


\section{RESULTADOS}

A análise de dados foi mediante a comparação dos resultados pré e pós teste, através do teste de equilíbrio, para saber a influência da modalidade Board Fitness na coordenação motora, por meio do equilíbrio corporal de mulheres adultas. Os dados foram apresentados por planilha no Excel, para serem comparados e então analisados para a conclusão da pesquisa.

Tabela 3. Dados comparativos da avaliação inicial e final.

\begin{tabular}{c|cccc} 
Identificação & $\begin{array}{c}\text { Idade } \\
\text { (anos) }\end{array}$ & $\begin{array}{c}\text { Avaliação } \\
\text { inicial (seg.) }\end{array}$ & $\begin{array}{c}\text { Avaliação final } \\
\text { (seg.) }\end{array}$ & Variação \\
\hline 1 & 24 & 2 & 8 & 6 \\
2 & 26 & 2 & 8 & 6 \\
3 & 26 & 2 & 6 & 4 \\
4 & 26 & 2 & 8 & 6 \\
5 & 26 & 2 & 6 & 4 \\
6 & 25 & 3 & 9 & 6 \\
7 & 22 & 2 & 8 & 6 \\
8 & 24 & 2 & 8 & 6 \\
9 & 21 & 6 & 10 & 4 \\
10 & 22 & 5 & 10 & 5
\end{tabular}

Fonte: elaborado pelo autor (2016)

Como pode-se observar na tabela 1, comparando-se os resultados das avaliações de equilíbrio corporal das 10 participantes há uma diferença nos resultados da avaliação inicial para a final, diante dos dados verifica-se uma melhora no equilíbrio corporal de todas as participantes. As participantes que conseguiram uma melhora menos significativa quando comparadas com outras participantes foi de 2 para 6 segundos, totalizando uma melhora de pouco mais de três segundos no equilíbrio corporal, enquanto os resultados mais expressivos foi de 3 para 9 segundos, uma melhora de 6 segundos no equilíbrio corporal em oito semanas de treinamento no board fitness. Tirando os centésimos que por critério do teste, contando somente números inteiros. Houve valores maiores de resultados, que foram de 10 segundos, porém a diferença da avaliação inicial para avaliação final é menor.

Tabela 4. Dados comparativos da média na avaliação pré e pós treinamento.

\begin{tabular}{lcc}
\hline Variáveis & Resultados & \pm Desvio Padrão \\
\hline Idade (anos) & 24,2 & 1,93 \\
Peso (Kg) & 56,42 & 7,81 \\
Altura (m) & 1,60 & 0,09 \\
Avaliação Inicial & 2,6 & 1,48 \\
Avaliação Final & 8 & 1,37
\end{tabular}

Fonte: elaborado pelo autor (2016)

Observa-se na tabela 2 as médias de resultados das participantes em relação aos resultados da avaliação inicial e final. Na avaliação inicial, nota-se que a média foi de 2,6 segundos, considerado nível iniciante na classificação do equilíbrio corporal. Já na avaliação final as participantes tiveram em média 8 segundos de equilíbrio corporal, totalizando uma melhora de 
mais de 5 segundos no equilíbrio corporal, o que fizeram avançar para o nível intermediário na classificação.

\section{DISCUSSÃO}

Inicialmente, um dado que nos chama atenção é o resultado da avaliação inicial do equilíbrio corporal. Pois, as participantes em questão são ativas quanto a pratica de atividade física e o nível de equilíbrio corporal encontra-se no iniciante, ou seja, elas conseguem permanecer em equilíbrio estático em média de 2,6 segundos.

Esse dado tão expressivo, quanto os índices elementares de equilíbrio corporal nos remetem a questionar a falta de preocupação com a coordenação motora na fase adulta pelos programas de treinamento de exercício físico. Fica evidente neste resultado, a hipótese de que nesta fase da vida, a coordenação motora fica esquecida, e a finalidade do exercício físico nesta fase enfatiza outras questões como a estética e a melhora das capacidades fisiológicas.

O teste denominado "parada da cegonha", que avalia o equilíbrio corporal de adultos mostrou-se um instrumento interessante de avaliação que, de acordo com a modalidade de trabalho de centros de treinamento ou academias, poderia ser incorporado na avaliação física aplicada por estas instituições, como um instrumento de acompanhamento na melhora do equilíbrio corporal ${ }^{6}$. Entretanto, também se observa uma escassez de métodos de avaliação da coordenação motora na fase adulta. Isso torna-se uma demanda a partir da preocupação dos profissionais da área de Educação Física em considerar a coordenação motora na fase adulta.

Assim como observado no estudo desenvolvido ${ }^{7}$, cujos autores estudaram o método Board Fitness no ganho de flexibilidade e equilíbrio, mantendo-se as condições de treinamento semelhante ao aplicado neste trabalho, foi encontrado melhora significativa nos testes relacionados no equilíbrio. Embora os autores utilizaram outro tipo de teste para a avaliação do equilíbrio, permanece a observação de uma melhora de 16,7 segundos, variando de 25,3 segundos, no teste realizado antes do período de treinamento, para 42 segundos no teste após os treinos. Com esses dados e com o artigo discutido, pode-se constatar que o equilíbrio é um parâmetro que apresenta melhoras em diferentes modalidades de treinamento.

Comparando os dados obtidos no teste antes e após o treinamento, pode-se observar significativa melhora. A média do tempo que os participantes permanecem na posição do teste passou de 2,6 para 8 segundos, o que também indica um avanço na tabela de níveis, saindo de iniciante e indo para o intermediário. Quando no caso de menor evolução, observou-se uma variação de 2 para 6 segundos no teste de equilíbrio, enquanto que o melhor resultado avançou de 3 para 9 segundos, ou seja, mesmo sendo um grupo de participantes homogêneo, ainda foi possível registrar uma diferença de 9-6 segundos entre as participantes

\section{CONCLUSÃO}

A relevância deste estudo está em se preocupar com o desenvolvimento motor na fase adulta. O desenvolvimento motor é inerente aos seres humanos. E quando pensamos na constituição humana e na sua relação com o meio o comportamento motor torna-se mediação entre esses âmbitos. Uma vez negligenciada esse aspecto, as ações motoras começam a regredir no desenvolvimento e a relação do sujeito com meio começa a ficar menos saudável.

Sendo assim, esse estudo vem denunciar a falta de preocupação no desenvolvimento motor na fase adulta, de discutir os processos de treinamento físico, por meio do exercício física, por conta do aumento alarmante de adeptos a sua prática, como forma de sistematizar esse treinamento afim de promover também a melhoras qualitativas na coordenação motora.

Nesta direção, o treinamento Board Fitness pode ser uma metodologia que contribuiu para melhora na coordenação motora através do equilíbrio corporal estático nas mulheres, fazendo com que melhore o estilo de vida das pessoas na fase adulta. É notável que em curto tempo de 
treinamento os ganhos nos resultados de equilíbrio corporal, no que diz respeito à coordenação motora.

Isso mostra a importância da realização deste tipo de trabalho, valorizando a atuação do profissional que busca a melhora do condicionamento físico das pessoas, fazendo que o profissional da área da Educação Física pense sua atuação de forma integral, considerando como promoção de saúde por meio do exercício física o desenvolvimento da coordenação motora.

\section{REFERÊNCIAS}

1. MEINEL,K. Motricidade I: teoria da motricidade esportiva sob o aspecto pedagógico. Rio de Janeiro: Ao Livro Técnico, v.1, 1984.

2. GALLAHUE, D. L; OZMUN, J.C. Compreendendo o desenvolvimento motor: bebês, crianças, adolescentes e adultos. São Paulo: Phorte, 2 ed., 2003.

3. MORAES, K. C; CORADINI, S. A; JUNIOR, A.J.S. O método do treinamento funcional board fitness para ganho de flexibilidade e equilíbrio. ENAF Science, Ribeirão Preto, v. 10, n. 1, p.16-23, jun. 2015.2 Disponível em: <http://www.enaf.com.br/novosite/revista_cientifica/revista_ENAF_congresso_cientifico_2015.p df\#page=24>. Acesso em: 26 abr. 2016.

4. BENELLI, M. Apostila Board Fitness, Campinas, 2014.

5. GUEDES, D.P; LOPES, C.C; GUEDES, J.E.R.P. Reprodutibilidade e validade do Questionário Internacional de Atividade Física em adolescentes. Revista Brasileira de Medicina Esportiva, Londrina, v. 11, n. 2, p. 151-158, mar/abr. 2005, https://doi.org/10.1590/S1517-86922005000200011.

6. PEREIRA, Cássio Adriano; DOMINGUES FILHO, Luiz Antonio. Efeito de um programa de exercícios proprioceptivos no equilíbrio postural de mulheres adultas saudáveis e fisicamente ativas. Coleção Pesquisa em Educação Física, Várzea Paulista, v.6, n.1, p.221-228, jul.2007. Disponível em: <http://www.editorafontoura.com.br/periodico/vol-6/Vol6n1-2007/Vol6n1-2007pag-221a228/Vol6n1-2007-pag-221a228.pdf>. Acesso em: 20 maio 2016.

7. CORADINI, S. A.; MORAES, K. C.; S. JUNIOR, A. J. O método do treinamento funcional board fitness para o ganho de resistência de força em membros superiores em adolescentes de 14 a 17 anos obesos e com sobrepeso. ENAF Science, Ribeirão Preto, v. 10, n. 1, p.24-30, jun. 2015. Disponível

em: http://www.enaf.com.br/novosite/revista_cientifica/revista_ENAF_congresso_cientifico_2015.pdf \#page=24>. Acesso em: 26 abr. 2016. 